\title{
Making measurements in the pulmonary circulation: when and how?
}

\author{
D Raeside, A Peacock
}

Primary pulmonary hypertension is a well known though rare condition, but pulmonary hypertension also occurs as a consequence of most heart and lung diseases where it is largely unrecognised and untreated and is therefore a major clinical challenge. The consequence of this failure to assess the pulmonary circulation is that the process may progress to the point where treatment is less likely to be beneficial. It is because the symptoms and signs of pulmonary hypertension are often rather vague and non-specific that it is important to be aware of methods of measurement in the pulmonary circulation and their sensitivity, specificity, and relative merits. There is no easily applied sphygmomanometer for the pulmonary circulation, but there are a number of techniques - both invasive and non-invasive which can help us to make the diagnosis. Implicit in the desire to make an earlier diagnosis of pulmonary hypertension is the belief that there is some point in doing so - that is, that the condition is itself of significance and that we may be able to offer some additional treatment to the patient.

\section{When to consider secondary pulmonary hypertension?}

The causes of secondary pulmonary hypertension are: (1) chronic hypoxic lung disease including chronic airflow obstruction, fibrosing lung disease, and ventilatory failure due to primary chest wall dysfunction; (2) thromboembolic pulmonary hypertension; and (3) connective tissue disease, especially CREST syndrome.

\section{CHRONIC HYPOXIC LUNG DISEASE}

While a number of conditions can give rise to secondary pulmonary hypertension, hypoxic lung disease is the one most commonly encountered in clinical practice. The extent of the problem is difficult to define and is further complicated by the debate about the term "cor pulmonale" which is often used when patients with respiratory failure develop oedema. This expression implies dysfunction of the heart when, in fact, the fluid retention is probably renal in origin, though is associated with the pulmonary hypertension. For this reason many authors would prefer that the term is no longer used and that it should be replaced with "fluid retention in association with secondary pulmonary hypertension". ${ }^{1}$

There is controversy about the contribution of secondary pulmonary hypertension to the mortality and morbidity of chronic hypoxic lung disease, but the important variations in pulmonary artery pressure caused by exercise, sleep (due to alveolar hypoventilation), ${ }^{2}$ and episodes of respiratory failure secondary to acute infection are now accepted, although the latter have been shown to return to normal before becoming resistant to treatment as the disease progresses. ${ }^{3}$ Clearly these are difficult to measure routinely because of the difficulty in making reliable measurements of pulmonary haemodynamics repeatedly. The signs and symptoms of pulmonary hypertension are usually overwhelmed by those of chronic obstructive pulmonary disease (COPD), but pulmonary hypertension should be suspected in patients with persistent hypoxaemia $\left(\mathrm{PaO}_{2}<7.3 \mathrm{kPa}\right)$ who complain of deteriorating exercise tolerance in the face of static spirometric values, or in those who develop evidence of fluid retention. This can be a difficult clinical diagnosis and one American study noted that up to $30 \%$ of admissions to hospital with a diagnosis of congestive cardiac failure actually had fluid retention associated with pulmonary hypertension. ${ }^{4}$

There is little doubt that secondary pulmonary hypertension is an important factor in the morbidity and mortality of chronic hypoxic lung disease and other conditions. Quantifying pulmonary haemodynamics should be an important part of assessment of these patients and can assist us in future decisions about the success or otherwise of treatment directed both at the primary respiratory disease and at the pulmonary circulation itself. Clearly, the first therapeutic objective must be the treatment of the pulmonary condition, ${ }^{5}$ but we should be monitoring the effects of this treatment and the use of vasodilators, if appropriate, on the pulmonary circulation. ${ }^{6}$

THROMBOEMBOLIC PULMONARY HYPERTENSION

This diagnosis should always be considered in a patient with a past history of deep venous thrombosis or pulmonary embolus who subsequently develops unexplained symptoms of breathlessness. Where the patient presents soon after the event the diagnosis may seem obvious, but Moser ${ }^{7}$ has described the "honeymoon period" after pulmonary embolism where the patient's symptoms can improve for a period of months or years before deteriorating again, sometimes rapidly. Significantly, his group estimated that between $0.1 \%$ and $1.0 \%$ of all embolic patients go on to develop thromboembolic pulmonary hypertension, and that in nearly half the initial event was unrecognised.

\section{CONNECTIVE TISSUE DISEASE}

Pulmonary vasculitis is rare in most connective tissue disorders, being seen in $<2 \%$ of patients with systemic lupus erythematosus, but it is a serious complication. ${ }^{8}$ In the CREST variant of systemic sclerosis severe pulmonary hypertension is much more common and is the most important cause of mortality and morbidity in this condition. Here the medium and small pulmonary arteries undergo pathological changes similar to those seen in primary pulmonary hypertension. Patients may present with minimal or no clinical respiratory signs other than breathlessness, but a clue is a low carbon monoxide gas transfer factor in the face of normal spirometric values.

\section{How to measure the pulmonary circulation?}

NON-INVASIVE METHODS OF MEASUREMENT

Traditional methods of assessment include tests which suggest the presence of pulmonary hypertension (clinical examination, electrocardiography, chest radiography, and conventional echocardiography) and those which suggest a cause (pulmonary function tests, ventilation perfusion scanning, and arterial blood gas tensions). These have re- 
cently been augmented by techniques which tell us about the severity of pulmonary hypertension, especially Doppler echocardiography which will be considered in more detail. However, the traditional methods are still of value.

Radiological assessment of pulmonary artery diameter, M mode echocardiography, and electrocardiography were compared in one study ${ }^{9}$ and each was found to have a high specificity for detection of pulmonary hypertension (particularly at higher levels of pulmonary hypertension) but were individually too insensitive to be useful for routine clinical screening; a normal test does not exclude pulmonary hypertension. More recent work ${ }^{10}$ has employed complex data analyses to assess the combined value of these tests, including forced expiratory volume in one second $\left(\mathrm{FEV}_{1}\right)$, arterial blood gas tensions, electrocardiography, pulmonary arterial dimension on chest radiography, $M$ mode echocardiography, and myocardial thallium scanning, and confirmed that this approach allows the prediction of the presence of pulmonary hypertension but gives no indication of the actual value of the pulmonary artery pressure.

Ventilation/perfusion lung scanning is widely used in the evaluation of patients with pulmonary hypertension, primarily to exclude thromboembolic disease and to assess the need for pulmonary angiography. Pulmonary function tests may likewise point to an underlying cause for suspected pulmonary hypertension such as COPD. Furthermore, an isolated reduction in diffusing capacity may be a pointer to pulmonary hypertension in a breathless patient with normal spirometric values.

\section{DOPPLER ECHOCARDIOGRAPHY}

The techniques described above, most of which remain in routine clinical use, can detect pulmonary hypertension but give no assessment of its severity. Hence, they cannot be used to follow the clinical course of patients with pulmonary hypertension or assess the benefits of intervention.

In 1984 Yock and Popp ${ }^{11}$ described a technique which allowed estimation of pulmonary arterial pressure from the measurement of the maximum velocity of the regurgitant jet in patients with tricuspid regurgitation (TR). When TR is present the velocity of the regurgitant jet is determined solely by the pressure difference between the right ventricle (RV) and the right atrium (RA) and not by the geometry of the orifice. To make this estimate the TR jet is identified and located using pulsed or colour Doppler before continuous wave Doppler recordings are made to determine peak TR jet velocity. Providing that the angle between the ultrasound beam and the direction of flow is less than 20 degrees, velocity of flow can be calculated accurately. This maximum velocity can then be used in a modified form of the Bernouilli equation which states that:

$\mathrm{RV}$ to $\mathrm{RA}$ pressure gradient $=$

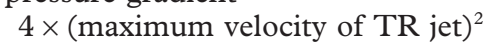

The calculated gradient is added to the right atrial pressure (which can be estimated clinically) to give an estimated value of right ventricular systolic pressure. Assuming there is no pulmonary stenosis, which can be excluded by echocardiography, this value will be equal to pulmonary arterial pressure. The authors have shown these values to correlate closely with those found at right heart catheterisation even when not measured simultaneously.

This technique confers a number of advantages. The values obtained are accurate over a wide range of pressures $^{12-14}$ and are not affected by changes in cardiac output. ${ }^{12}$ Interobserver variability is $<3 \%,{ }^{12}$ though clearly the technique is, to some extent, operator dependent.
Continuous Doppler echocardiography has attained widespread use and recent data suggest that the error limits for Doppler TR derived pressure calculations are only 5-9 mm Hg. ${ }^{15}$ The technique is well tolerated, safe, and repeatable, but there are some disadvantages. In particular, it is difficult to recover a TR signal of sufficient quality in patients with COPD where success rates may be as low as $30 \% .^{16}$

There is no such difficulty in examining flow characteristics in the right ventricular outflow tract. The pulsed Doppler signal is directed at the pulmonary valve and two important measurements are made - namely, acceleration time (AT) and right ventricular ejection time (ET). ${ }^{17}$ AT is defined as the time from the onset of ventricular ejection to the attainment of maximum flow velocity through the pulmonary valve, and ET is defined as the interval between the beginning and end of flow in the right ventricular outflow tract. These flows have characteristic pulsed wave Doppler velocity envelopes. Normal subjects have a smooth dome-shaped envelope while patients with pulmonary hypertension show a notched pattern caused by a shortened AT followed by a second slower rise during deceleration. AT and ET can therefore be used to demonstrate the presence of pulmonary hypertension but, unlike continuous wave Doppler assessment of TR jet velocity, the calculation of underlying values of pulmonary artery pressure is poor. ${ }^{18}$ Intra and interobserver variability is slightly worse than with continuous wave Doppler and the technique is less sensitive to factors which induce acute changes in pulmonary artery pressure. ${ }^{19}$ However, compared with the success rate for continuous wave Doppler in COPD of approximately $30 \%$, pulsed wave Doppler can detect pulmonary hypertension in over $90 \%$ of cases.

\section{INVASIVE METHODS OF MEASUREMENT}

Conventionally, fluid filled thermodilution catheters have been taken as the gold standard against which other techniques have been compared, though this view is not accepted by some authors. ${ }^{20}$ Right heart catheterisation is usually carried out using a fluid filled thermodilution catheter of the Swan Ganz type. ${ }^{21}$ The procedure can be used to answer a number of questions and is very fully described elsewhere ${ }^{22}$ but, essentially, the catheter is inserted via a central vein and advanced until the tip is placed in the pulmonary artery. Position can be determined either from the pressure trace or by direct screening with fluoroscopy. The catheter has two fluid filled lumina from which pressure traces of right atrial and pulmonary artery pressures are recorded simultaneously by external transducers, and a thermistor for measurement of pulmonary blood flow by the thermodilution technique. Pressure measurements are conventionally made in the right atrium, right ventricle, pulmonary artery, and in the wedge position to record pulmonary artery occlusion pressure which is an estimate of left atrial pressure. Pressure and flow measurements can be repeated following interventions such as the administration of vasodilators or oxygen, or after exercise. Thus, in addition to the direct measurement of pressures, a number of variables are derived which can be used to calculate further information such as pulmonary vascular resistance (PVR) which is calculated by subtracting the pulmonary artery occlusion pressure (estimated left atrial pressure) from the mean pulmonary artery pressure and dividing by the cardiac output. This value of PVR (expressed in Wood's units) may be altered by the interventions mentioned above to give an idea of the vasoreactivity of the pulmonary circulation.

As with the other investigative tools discussed so far there are advantages and disadvantages to right heart catheterisation, but most important are the significant risks to 
the patient ranging from the common and relatively trivial, such as self limiting ventricular arrhythmias, to the very rare but often fatal rupture of the pulmonary artery (risk approximately 1/3000). ${ }^{23}$ Apart from the risks, hospital admission is usual which may be inconvenient for patients. There are also problems inherent in the technique. The fluid filled catheter acts as a damper of the signal and is incapable of measuring instantaneous pressures. Furthermore, these catheters rely on an external transducer which is levelled with the right atrium making it impossible for the patient to change position and allowing limited manoeuvres on the catheter table. The measurements are made in unrepresentative surroundings (the catheter laboratory) in an unrepresentative position (lying supine on a table) and in an unrepresentative state (resting). Hence the results obtained, though of value, bear little relation to the pulmonary haemodynamics actually experienced by patients.

Micromanometer tipped, high fidelity catheters overcome some of these problems. They are solid catheters with the reference transducer located in the tip which allows the patient to be ambulant. However, there are problems inherent in this design. These catheters are subject to zero drift - that is, spontaneous variation in recorded baseline pressure. Though this zero drift may have very small actual values, these can be very important when the pressures being measured are low. ${ }^{24}$ Consequently, newer micromanometer tipped catheters have been developed with the pressure transducer connected to an in vivo calibration system to correct zero drift. When used with the relevant recording and information storage systems, these catheters allow the accurate recording of long term (>24 hours) pulmonary pressures..$^{25}$ These, when analysed in conjunction with a simple patient diary, reveal changes in pulmonary artery pressure with everyday activity such as sleeping, standing, walking, or exercising. ${ }^{26}$ More formal exercise testing can also be carried out with the catheter in situ. However, though superior to fluid filled catheters in many respects, micromanometer tipped catheters are expensive, less flexible, and lack a flotation balloon which can lead to difficulties in placement. Since the patient should be ambulant to gain maximum information, the femoral route is not possible and the internal jugular or subclavian route must be used.

Despite their advantages, neither type of pulmonary artery catheter takes into account all the complex forces which constitute the pulmonary haemodynamics. However, when combined with Doppler echocardiography a much more complete picture is obtained.

In conclusion, there is now a formidable array of tools available for the investigation of a patient with suspected pulmonary vascular abnormalities. Though the more traditional methods have been shown to be too insensitive to be valuable in isolation, collectively they remain important screening techniques by which patients who require further investigation can be identified.

Pulmonary hypertension is an important complication of many cardiorespiratory diseases and its recognition is important. When it is suspected, usually on the basis of the patient's signs and symptoms and the traditional screening tests, referral should be made, in the first instance, for echocardiography. This should include assessment by both continuous wave and pulsed wave Doppler. When pulmonary hypertension is confirmed, or where there continues to be any doubt, the patient should be referred to a pulmonary vascular centre for further assessment.

\section{Correspondence to: Dr A Peacock.}

Pulmonary Vascular Unit,

Department of Respiratory Medicine,

D RAESIDE

Western Infirmary,

West Glasgow Hospitals University NHS Trust,

Glasgow G11 6NT, UK

1 Weitzenblum E, Chaouat A, Oswald M. Pulmonary hypertension due to chronic hypoxic lung disease. In: Peacock AJ, ed. Pulmonary circulation, chronic hypoxic lung disease. In: Peacock AJ, ed. Pulmonary circulation,

2 Boysen PG, Block AJ, Wynne JW, Hunt LA, Flick MR. Nocturnal pulmonary hypertension in patients with chronic obstructive pulmonary disease. $\mathscr{F}$ Appl Physiol 1979;54:632-9.

3 Weitzenblum E, Loiseau A, Hirth C, Mirhom R, Rasaholinjanahary J. Course of pulmonary haemodynamics in patients with chronic obstructive pulmonary disease. Chest 1979;75:656-62

4 Intersociety Commision for Heart Disease Resources. Primary prevention of pulmonary heart disease. Circulation 1970;41:A17-23.

5 Peacock AJ. Pulmonary hypertension due to chronic hypoxia: treat the lung not the pressure. BMF 1990;300:763.

6 Peacock A. Vasodilators in pulmonary hypertension. Thorax 1993;48:11969.

7 Moser KM, Fedullo PF. Acute and chronic pulmonary thromboembolism. In: Peacock AJ, ed. Pulmonary circulation, a handbook for clinicians. London: Chapman and Hall, 1996:392-422.

8 Gross M, Esterly JR, Earle RH. Pulmonary alterations in systemic lupus erythematosus. Am Rev Respir Dis 1972;105:572-7.

9 Algeo S, Morrison D, Ovitt T, Goldman S. Non-invasive detection of pulmonary hypertension. Clin Cardiol 1984;7:148-56.

10 Bishop JM, Csukas M. Combined use of non-invasive techniques to predict pulmonary arterial pressure in chronic respiratory disease. Thorax 1989 44:85-96.

11 Yock P, Popp R. Non-invasive estimation of right ventricular systolic pressure by Doppler ultrasound in patients with tricuspid regurgitation. Circulation 1984;70:657-62.

12 Berger M, Haimowitz A, Van Tosh A, Berdoff RL, Goldberg E. Quantitative assessment of pulmonary hypertension in patients with tricuspid regurgitation using continuous wave Doppler ultrasound. $\mathcal{F}$ Am Coll Cardio 1985;6:359-65.

13 Currie PJ, Seward JB, Chan KL, Fyfe DA, Hagler DJ, Mair DD, et al. Continuous wave Doppler determination of right ventricular pressure: a simultaneous Doppler-catheterisation study in 127 patients. $\mathcal{f} \mathrm{Am}$ Coll Cardiol 1985;6:750-6.

14 Chan KL, Currie PJ, Seward JB, Hagler DJ, Mair DD, Tajik AJ. Comparison of three ultrasound methods in the prediction of pulmonary artery pressure. f Am Coll Cardiol 1987;9:549-54.

15 Naeije R, Torbicki A. More on the non-invasive diagnosis of pulmonary hypertension: Doppler echocardiography revisited. Eur Respir $\mathcal{f}$ 1995;8: $1445-9$

16 Torbicki A, Skwarski K, Hawrelkiewicz I, Pasierski T, Miskiewicz Z, Zielinski J. Attempts at measuring pulmonary artery pressure by means of Doppler echocardiography in patients with chronic lung disease. Eur Respir 71989 2:856-60

17 Kitabatake A, Inoue M, Asao M, Masuyama T, Tanouchi J, Morita T, et al. Non invasive evaluation of Doppler by a pulsed wave technique. Circulation 1983;68:302-9.

18 Tramarin R, Torbicki A, Marchandise B, Laaban JP, Morpurgo M. Doppler echocardiographic evaluation of pulmonary artery pressure in chronic obstructive pulmonary disease. A European multicentre study. Eur Heart f 1991;12:103-11.

19 Torbicki A, Tramarin R, Fracchia C. Reliability of pulsed wave Doppler monitoring of acute changes in pulmonary artery pressure in patients with COPD. Prog Respir Res 1990;26:133-41.

20 Naeije R. Pulmonary vascular function. In: Peacock AJ, ed. Pulmonary circulation, a handbook for clinicians. London: Chapman and Hall, 1996: 13-27.

21 Swan HJC, Ganz W, Forrester JS, Marcus H, Diamond G, Chonnette D. Catheterisation of the heart in man with the use of a flow directed catheter. N Engl F Med 1970;283:447-51.

22 Groves BM, Badesch DB. Cardiac catheterisation of patients with pulmonary hypertension. In: Peacock AJ, ed. Pulmonary circulation, a handbook for clinicians. London: Chapman and Hall, 1996:51-67.

23 Kearney TJ, Shabot MM. Pulmonary artery rupture associated with the Swan Ganz catheter. Chest 1995;108:1349-52.

24 Gibbs JSR, Cunningham D, Sparrow J, Poole-Wilson PA, Fox MK. Unpredictable zero drift in intravascular micromanometer tipped catheters during long term pulmonary artery pressure recording: implications for catheter design. Cardiovasc Res 1989;23:152-8.

25 Gibbs JSR, MaClachlan D, Fox KM. A new system for ambulatory pulmonary artery pressure recording. Br Heart $\mathcal{f}$ 1992;68:230-5.

26 Chalmers G, Bicknell SR, Peacock AJ. 24 hour ambulatory pressure measurements in patients with connective tissue disease: relation to posture and activity (abstract). Am F Respir Crit Care Med 1996;53:4,2/2. 